\title{
Global Gene Expression in the Developing Rat Brain After Hypoxic Preconditioning: Involvement of Apoptotic Mechanisms?
}

\author{
MALIN GUSTAVSSON, MARY ANN WILSON, CARINA MALLARD, CATHERINE ROUSSET, MICHAEL V. JOHNSTON, \\ AND HENRIK HAGBERG
}

\begin{abstract}
Departments of Physiology [M.G., C.M., C.R., H.H.] and Obstetrics and Gynecology [H.H.], Sahlgrenska Academy, Göteborg University, 41685 Göteborg, Sweden; Departments of Neurology [M.A.W., M.V.J.], Neuroscience [M.A.W.], and Pediatrics [M.V.J.], Kennedy

Krieger Research Institute, Johns Hopkins University School of Medicine, Baltimore, Maryland 21205
\end{abstract}

\begin{abstract}
Exposure to hypoxia before hypoxia-ischemia (HI) confers substantial protection referred to as preconditioning (PC). We hypothesized that PC induces critical changes of genes related to apoptotic cell death to render the brain more resistant. PC hypoxia $\left(8 \% \mathrm{O}_{2}, 36^{\circ} \mathrm{C}, 3 \mathrm{~h}\right)$ was induced in rats on postnatal day (PND) 6 , and the rats were killed at $0,2,8$, and $24 \mathrm{~h}$. Total RNA was extracted from cerebral cortex and analyzed using Affymetrix rat genome 230 2.0 array. PC induced significant changes in 906 genes at 0 h, 927 at $2 \mathrm{~h}, 389$ at $8 \mathrm{~h}$, and 114 at $24 \mathrm{~h}$. Ontology analysis revealed significant alterations in genes involved in cell communication, signal transduction, transcription, phosphorylation, and transport. Genes involved in cell death/apoptosis as well as those related to brain development (cell differentiation, neurogenesis, organogenesis, blood vessel development) were overrepresented. A detailed analysis demonstrated that 77 significantly regulated genes were involved in apoptosis, specifically related to the Bcl-2 family, JNK pathway, trophic factor pathways, inositol triphosphate (PI3) kinase/Akt pathway, extrinsic or intrinsic pathway, or the p53 pathway. The study supports that the epidermal growth factor receptor family, mitogenactivated protein kinase phosphatases, and Bcl-2-related proteins and the PI3 kinase/Akt pathway may have roles in providing resistance in the developing central nervous system (CNS). (Pediatr Res 61: 444-450, 2007)
\end{abstract}

$\mathrm{I}_{\mathrm{i} / \mathrm{m}}^{\mathrm{m}}$ njury in the developing brain is a common cause of disability in children. Although multiple factors such as excitotoxicity, reactive oxygen species, inflammation, and apoptotic mechanisms have been shown to be involved, there are no effective treatments at this time (1), and there is a need for increased knowledge regarding the pathophysiology of perinatal brain damage. Exposure to sublethal hypoxic conditions (hypoxic PC) $24 \mathrm{~h}$ before HI confers substantial protection of the developing brain (2). We have recently observed that reduction in brain injury is long-lasting ( $8 \mathrm{wk}$ ) with respect to both histopathology and long-term sensorimotor behavioral tasks (3). There are reasons to believe that altered gene

Received May 2, 2006; accepted December 6, 2006.

Correspondence: Henrik Hagberg, M.D., Ph.D., East Hospital, 41685 Göteborg, Sweden; e-mail: henrik.hagberg@obgyn.gu.se

This work was supported by NIH-NINDS grant R01 NS 28208 (MVJ), the Swedish Medical Research Council 09455 (HH), and Swedish governmental grants to scientists in working in health care (ALFGBG-2863) (H.H.).

Supplemental material available on the Pediatric Research Web site at www. pedresearch.org.

DOI: 10.1203/pdr.0b013e3180332be4 expression underlies this protective effect, e.g. a certain time is needed for induction of the lowered CNS vulnerability and inhibitors of gene transcription/protein synthesis block PC (4). We hypothesized that characterization of alterations in the global gene expression after PC would give important clues to mechanisms of induced tolerance in the developing brain and may identify targets for therapeutic intervention (5).

There is one such study previously published using the microarray chip Affymetrix U34A (contained approximately 8000 genes/expressed sequence tags) that provides interesting information (6), but still there is a scarcity of data on how apoptotic genes are altered by hypoxic PC. Indeed, several lines of evidence support that apoptotic mechanisms are center stage in cerebral vulnerability in response to $\mathrm{HI}$ insult in neonates. Key elements of apoptosis have been demonstrated to be strongly up-regulated in the immature brain, such as caspase-3 (7), APAF-1 (8), Bcl-2 (9), and Bax (10). Caspase-3 is markedly activated after $\mathrm{HI}$ in the immature brain (11-14), and cells with the cleaved active form of caspase- 3 colocalize with markers of DNA fragmentation in injured brain regions (15). Caspase-3 inhibitors (11) as well as transgenic overexpression of X-linked inhibitor of apoptosis (XIAP) (16) attenuate caspase-3 activation and provide neuroprotection. Assembly of the apoptosome is easily induced in homogenates from the immature (but not adult) brain (17), cytochrome $c$ is released to the cytosol in response to $\mathrm{HI}(15,18)$, and caspase-9 is activated. In addition, other proapoptotic proteins like AIF (14), SMAC/Diablo (16), and HtrA2/Omi (16) translocate from the mitochondria to the nucleus, suggesting that proapoptotic proteins are indeed released during the early recovery phase after $\mathrm{HI}$.

In the present study, we characterize global gene expression after hypoxic PC using a recently introduced gene chip containing approximately 31,000 genes/ESTs with the specific aim to identify novel genes that may be involved in the apoptotic cascade and that could contribute to rendering the brain more resistant to HI. In parallel experiments, we confirmed that the PC insult used here indeed provided protection against HI.

Abbreviations: PC, preconditioning; HI, hypoxia-ischemia; PND, postnatal day 
Table 1. Summary of the number of genes significantly ( $t$ test, FDR 0.05, 1.2-fold, BH correction) up- or down-regulated 0, 2, 8, and $24 \mathrm{~h}$ after $P C$

\begin{tabular}{lcc}
\hline Time point & No. up-regulated & No. down-regulated \\
\hline $0 \mathrm{~h}$ & 645 & 261 \\
$2 \mathrm{~h}$ & 691 & 236 \\
$8 \mathrm{~h}$ & 178 & 211 \\
$24 \mathrm{~h}$ & 79 & 35 \\
\hline
\end{tabular}

\section{MATERIALS AND METHODS}

Experimental animals. Sprague-Dawley rats, originating from Charles River Laboratories (Sulzfeld, Germany), were bred at Göteborg University local animal care facility EBM (Göteborg, Sweden). Animals were housed in accordance to standard guidelines. The experimental protocol was approved by the Regional Animal Ethical Committee of Göteborg (no. 293-01).

Hypoxic PC. On PND 6, animals $(n=64)$ were subjected to PC hypoxia for $3 \mathrm{~h}\left(8.0 \%\right.$ oxygen in nitrogen, $\left.36^{\circ} \mathrm{C}\right)$. Sham controls $(n=56)$ drawn from the same litters were simultaneously exposed to normoxia $\left(36^{\circ} \mathrm{C}\right)$. Further, animals were either used for RNA preparation followed by microarray analysis or for confirmation of neuroprotection with respect to HI at PND 7.

RNA preparation. Animals were killed and brains collected at four time points $(0,2,8$, and $24 \mathrm{~h})$ after the $\mathrm{PC} /$ sham procedure. Five brains per group were collected at each time point, with an equal number of males and females in each group. Brains were removed and dissected on ice, and the cerebral cortex of both hemispheres was rapidly frozen on powdered ice. Total RNA from cerebral cortex was isolated using an RNeasy Protect Maxi kit, according to the manufacturer's instructions (Qiagen Inc.). RNA was quantified by spectrophotometry at $260 \mathrm{~nm}$, and the OD determined by a 260/280 ratio. The quality of the RNA was further checked by running the RNA samples on a $1.1 \%$ agarose $/ 2.2 \mathrm{M}$ formaldehyde gel to ascertain that there was no degradation.
Microarray analysis. Microarray analysis was performed at the National Institutes of Health Neuroscience consortium for microarray analysis (http:// arrayconsortium.tgen.org/np2/home.do), using one array for each animal. There were five animals per treatment group ( $\mathrm{PC}$ or sham) at each time point $(0,2,8$, and $24 \mathrm{~h})$, for a total of 40 arrays. We used the Affymetrix 2302.0 array, which comprises more than 31,000 probe sets, analyzing more than 30,000 transcripts and variants from 28,000 well-substantiated rat genes. In addition to PubMed, the following databases were used to obtain information about specific genes of interest: http://copewithcytokines.de/ and http:// genome-www5.stanford.edu/cgi-bin/source/sourceSearch.

The raw cell average intensity (CEL) and derived chip (CHP) data, as well as minimum information about a microarray experiment (MAIME)-compliant project information are available for download through the National Institutes of Health Neuroscience Microarray Consortium (http://arrayconsortium.tgen. org/np2/navigateRepository.do; Project: wilso-affy-rat-132990).

Statistical analysis. Data management, normalization, statistical analysis, and gene ontology (GO) analysis were performed using the Web-based GeneSifter software (http://www.genesifter.net). Affymetrix CEL files were preprocessed using the robust multiarray average (RMA) algorithm $(19,20)$, which performs three distinct operations: global background normalization, across-array normalization, and $\log _{2}$ transformation of perfect match values (http://stat.www.berkeley.edu/users/bolstad/RMAExpress/RMAExpress. $\mathrm{html}$ ). All 40 arrays were normalized together as one experiment to reduce nonbiological variability. Changes in gene expression in the sham controls over the 24-h experimental period $(0,2,8$, and $24 \mathrm{~h})$ were analyzed using analysis of variance (ANOVA) (with $0 \mathrm{~h}$ set as control) with the Benjamini and Hochberg (21) false discovery rate (FDR) correction for multiple comparisons set at 0.05 and a threshold of 1.2-fold change for inclusion. Pairwise comparison of the 0- and 24-h time points in sham controls was carried out using a $t$ test with FDR set at 0.05 . Because there were significant changes in gene expression in sham controls over this $24 \mathrm{~h}$ period, the effect of PC gene expression was examined separately at each time point versus time-matched sham controls. At each time point, gene expression was analyzed using $t$ tests with the Benjamini and Hochberg (BH) FDR set at 0.05 and a threshold of 1.2 -fold change for inclusion. Further analysis of GO terms for those genes

Table 2. Gene ontology classification of genes that are significantly different versus sham at 0,2 , or 8 h after PC

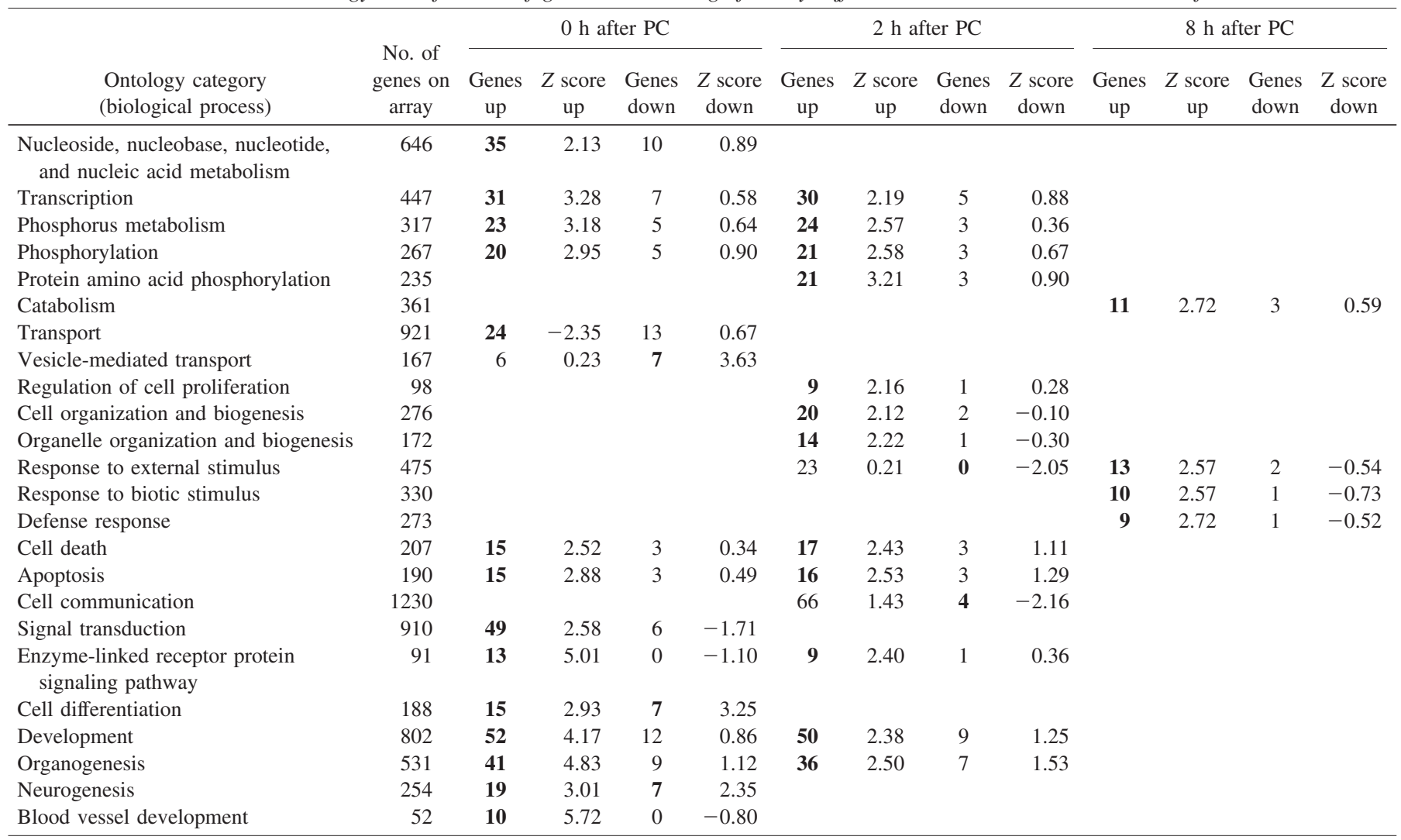

The number of genes up- or down-regulated is shown in bold type for biological process terms that occur more frequently $(Z$ score $>2)$ or less frequently $(Z$ score less than -2$)$ than expected by chance. Only terms including at least 10 genes and being significantly over- or underrepresented are shown.

Up, up-regulated; down, down-regulated. 
gene name(ID)

Metallothionein(Mt1a)

DNA-damage-induc, transer, 4(Ddit4)

Basic helix-loop-helix domain containing, class B2(Bhlhb2)

Bone morphogenetic protein 6(BMP6)

Activating transcription factor 3(Atr)

Trefoil factor 2 (Tff2)

Sunvival of motor neuron 1(Smn1)

Cysteine, glycine-rich protein 2(csrp2)

Neurotrophin 3 (ntt3)

Nuclear transcription factor-Ya(Nfya)

Arginine-glutamic acid dipeptide (RE) repeats(RERE)

CAMP responsive element modulator(CREM)

Growth arrest and DNA-damage-inducible 45b(GADD45b)

Serine/threonine kinase $17 \mathrm{~b}($ Stk17b)

SUMO/sentrin spec. protease 2(Senp2)

Tissue inhibitor of metalloproteinase (Timp) 3

Tissue inhibitor of metalloproteinase (Timp)2

Tissue inhibitor of metalloproteinase (Timp) 1

Nudix (nucleoside diphosphate linked moiety $X$ )-type moti(Nudt6)

Transducin-like enhancer of split 3, E(spl) homolog(Tle3)

Notch gene homolog 1(notch1)

6 protein-coupled receptor 37(Gpr37)

Zyxin(zyx)

Protein kinase N1(Pkn1)

Heat shock protein 2(HspA2)

Heat shock 7010 protein 18 (Hspa1a)

Tumor necrosis factor receptor superfamily $12 \mathrm{a}\left(\mathrm{Tnfrsf}_{12 \mathrm{a}}\right.$

Ret proto-oncogene(Ret)

Serine/threonine kinase 6 (StK6)

Tumor suppressor pHyde $(\mathrm{L} O \mathrm{C170824)}$

Caveolin (Cav)

Mitogen activated protein kinase kinase kinase 1(Map3k1)

Max interacting protein 1(Mxi1)

Inhibitor of DNA binding 1(ld1)

Notch gene homolog 2(Notch2)

Eyes absent 2 homolog (Eya2)

Ngfi-A binding protein 2(Nab2)

BC12-associated athanogene 3(Bag3)

BCL2/adenovirus E1B $19 \mathrm{kDa}$-interacting protein 3(Bnip3)

Pleiomorphic adenoma genelike1(Plagl1)

c-jun kinase 3 (JNK3/Mapk10)

Signal transducer and activator of transcription 3 (stat3)

B-cell translocation gene 2(Btg2)

Nuclear factor $\mathrm{k}$ light chain gene enhancer in B-cells inhibitor a(Nikbia)

Nuclear receptor subfamily 4 , group A, member $1(\mathrm{Nr} 4 \mathrm{a} 1=\mathrm{Nur} 77)$

LPS-induced TNF-alpha factor(Litaf)

Jun-B oncogene(Junb)

Basic helix-loop-hellx domain containing, class B3(Bhlhb3)

Dual specificity phosphatase 5(Dusp5)

Dual specificity phosphatase 1(Dusp1)

Hairy and enhancer of split 1(Hes 1)

Neuregulin 1(Nrg-1)

Insulin-like growth factor 1 rec(lof 1n)

CCAAT/enhancer binding protein (CIEBP) b(Cebpb)

Insulin-like growth factor binding protein 3(lgfbp3)

Serum/glucocorticoid reg. Kinase(sgk)

Heme oxygenase-1(Hmox 1$)$

Early growth response 2(Egr2)

Early growth response 1(Egr1)

\section{functional summary}

Heavy metal binding.anti-apoptosis and anti-inflammation mediates anti-apoptotic PI3 kinase \& HIF-1-dependent signals inhibits activation of the mitochondrial pathway of apoptosis anti-ischemic anti-apoptotic in adult brain

induces HSP27 \& AKt which block JNK-dependent apoptosis protective factor in the gut and anti-apoptotic effects in cancer cells anti-apoptotic effects in neuronal cells, caspase dependent binding partner of inhibitor of STAT-1 involved in CNS apoptosis anti-apoptotic action via activation of AKt in CNS involvement in p53-induced GADD45 triggered apoptosis ? interacts with BAX and affects caspase-dependent apoptosis pro- or anti-apoptotic effects via CAMP and CREB pathways blocks JNK- and Fas-mediated apoptosis through NFKB apoptosis-inducing kinase in several tissues(=DRAK2) peptidase that regulate beta-catenin and o-jun + or - on apoptosis anti-inflammatory and induces apoptosis in inflammatory cells anti-inflammatory and induces apoptosis in inflammatory cells anti-inflammatory and induces apoptosis in inflammatory cells anti-sense for bFGF which has anti-apoptotic effects groucho-like protein that modulates Notch and apoptosis promotes survival of neural precursor cells via bcl-2 and mcl-1 enhances substantia nigra neuronal susceptibility anti-inflammatory and pro-apoptotic in inflammatory cells ? involved in TRAF2NFKB regulation of inflammation and apoptosis HSP7O family, ant-apoptotic, multiple pathways e.g. Bcl-2 anti-apoptotic effects in CNS

activates NFKB, ERK and JNK pathways, decoy reduces stroke injury receptor that activates PI3 kinase/AKt anti-apoptotic effects phosphorylates p53 at two sites with + or - effect in apoptosis induction of apotosis

induces Alk and exerts anti-apoptotic effects

control of JNK, ERK and NFKB with + or - effects on apoptosis HIF-1 dependent supression of c-myc induced apoptosis pro-apoptotic effect via production of reactive oxygen species in heart anti-apoptotic effect and promote cell survival in medulloblastomas pro-apoptotic effect via bcl-2 familiy proteins but not caspases represses Egr-1 which induces neuronal apoptosis anti-apoptotic bcl-2 familiy protein interacting with HSPs(predicited) pro-apoptotic BH3-only Bcl-2 family member and involved in autophagy pro-apoptotic (stim. p53-induced Apaf-1)and stimulates plasticity pro-apoptotic playing a key role in cell death after hypoxia-ischemia anti-apoptotic,JAK-STAT pathway, partly via BCl-2 anti-apoptotic, interacts with $p 53$ and SMADs inhibits NFKB mediated cell death and inflammation regulator with +1 - effect on apoptosis dep. on subcellular localization gene associated with apoptosis and TNF-a signaling component of AP-1 regulating proliferation and apoptosis regulates proliferation and apoptosis substrate of p53 and inhibits JNK3, p38 and Erk1/2 kinases substrate of p63 and inhibits JNK3, p38 and Erk1/2 kinases anti-apoptotic effect related to Notch pathway

anti-inflammatory and anti-apoptotic via Erb receptors anti-apoptotic via PI3KJAKt

anti-apoptotic gene acting through unknown mechanisms pro-apoptotic effect via p53 anti-apoptotic effect mediated via PI3K pathway protective anti-apoptotic effect in liver pro-apototic effect via Bcl-2 family proteins pro- or anti-apoptotic but mostly pro reported
Fold change PC vs. Control

REF novel access. \# Oh $2 \mathrm{~h} \quad 8 \mathrm{~h} \quad 24 \mathrm{~h}$

\begin{tabular}{|c|c|c|c|c|}
\hline a) & AF 411318 & A 2.8 & $\$ .3 .2$ & A 1.9 \\
\hline $1^{*}$ & NM_080906 & A 2.9 & A. 1.4 & \\
\hline $2^{*}$ & NM_053328 & ^ 2.1 & & \\
\hline $3^{*}$ & AW141680 & 个 1.2 & & \\
\hline 4 b) & NM_012912 & A 1.2 & & \\
\hline $5^{*}$ & NM_053844 & $\Downarrow 1.2$ & & \\
\hline $6^{*}$ & NM_022509 & $\Downarrow 1.2$ & & \\
\hline
\end{tabular}

$7^{*} \quad$ U44948 $\vee 1.2 \vee 1.3$

8 * NM_031073 A 1.2

$9^{*} \quad$ AW141680 $\downarrow 1.2$

$10^{*}$ Al172033 A. 1.2

$11.12^{*} \quad$ NM_017334 A 1.2

13.14 * B1287978 个. 1.2

$15^{*} \quad \mathrm{~A} 1012590 \quad \downarrow 1.2 \vee 1.2$

$16.17^{*}$ AA997863 A 1.2

$18^{*} \quad$ NM_012886 A 1.2 ^ 1.3

$18^{*}$ BF523128 ^ 1.2 ^ 1.2

$18 \mathrm{c})$ NM 053819 ^ 1.4 ^ 1.4

$19^{*} \quad$ U58289 $\quad \downarrow 1.2$

$20^{*} \quad$ NM_053400 A 1.2

$21^{*} \quad 8 M 390614$ 个 1.2

22 * NM_057201 $\downarrow 1.2$

23 * AA943537 A 1.2

$24^{*}$ AA945828 A 1.2

$25 *$ d) BF410146 A 1.2

26 *d) NM_031971 $\uparrow$. 1.2 ^.1.8

$27^{*} \quad 81303379$ 个 1.2 \& 1.3

28 * Al639318 $\uparrow 1.3 \uparrow 1.3$

$29^{*} \quad$ AA996882 $\vee 1.3$

* AF335281 $\vee 1.3$

30 a) AW915173 $\$ 1.3 \$ 1.4$

*e) Al102620 ^ 1.3

31 *f) A1409308 A 1.3

32 * M86708 A 1.3

$33^{*} \quad \mathrm{~A} 1011448$ 个 1.3 \$. 1.3

34 * BF386078 ^ $1.3 \uparrow 1.3$

35.36 * Al102530 क 1.3

$37^{*}$ A1231792 A. 1.3 ^.1.5

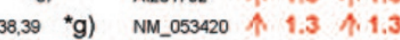

40,41 *h) $8 F 396790$ 个 1.3

42 * BF404073 ^ 1.3

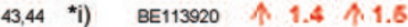

$45,46 * j) \quad$ NM_017259 ^ 1,4

$47^{*} \quad$ AW672589 A $1.4 \uparrow 1.5$

48 *k) NM_024388 ^ $1.4 \$ 1.3$

$49^{*} \quad 81284739$ 个 $1.4 \uparrow 1.3$

50 *l) NM_021836 A 1.4

$\left.51^{*} \mathrm{~m}\right) \quad$ NM_133303 个 1.4

52 * NM_133578 A. 1.4 ^.1.3

52 * BE110108 A 1.6

53 * NM_024360 个 1.4

54 * U02323 个 1.5

$55^{*} \quad$ A1044666 A 1.5

56 * NM_024125 $\uparrow 1.5 \propto 1.3$

$\left.57^{*} \mathrm{n}\right) \quad$ NM_012588 $\uparrow \mathrm{i} 1.5 \propto 1.7$

58 *o) NM_019232 $\uparrow 1.5 \$ 1.4$

$\left.59{ }^{*} \mathrm{p}\right) \quad$ NM_012580 $\uparrow 1.6 \% 1.4$

$60^{*} \quad$ NM_053633 $\uparrow 1.6 \propto 1.8$

$\left.61^{*} \mathrm{q}\right)$ NM_012551 $\uparrow 2.0 \uparrow 1.7$

Figure 1. Genes involved in apoptotic cell death.

that were significantly up- or down-regulated used a $Z$ score method to identify biologic process terms that occur more frequently or less frequently than expected ( $Z$ score $>2$ or less than -2 , respectively). Analysis of macroscopic neuropathological scores and reverse transcriptase polymerase chain reaction (RT-PCR) results was performed using the Mann-Whitney $U$ test, with significance at $p<0.05$.

Real-time PCR. To verify results of the microarray analysis, real-time RT-PCR (LightCycler, Roche Diagnostics) was used to examine mRNA expression for Ddit4 (NM_080906), Bhlhb2 (NM_053328), map3k1 (AI102620), Litaf (BI284739), Tnfrsfla (NM_013091), Atf3 (NM_012912), and Dusp1 (BE110108) at $0 \mathrm{~h}$, which (except for Tnfrsfla) were significantly regulated according to the microarray analysis. First-strand cDNA was syn- thesized from the same total RNA used for the microarray preparations with the Superscript RNase H reverse transcriptase kit (Life Technologies, Inc.) using random hexamer primers as previously described (7). Each $20 \mu \mathrm{L}$ contained $1 / 400$ of the cDNA synthesis reaction; $3 \mu \mathrm{M} \mathrm{MgCl} 2$, which was found to be the optimal concentration for each primer pair; $0.5 \mu \mathrm{M}$ forward and reverse primers; and $2 \mu \mathrm{L}$ Lightcycler FastStart DNA Master SYBR Green I (Roche Diagnostics). The following primer pairs (CyberGene AB, Huddinge, Sweden) annealing temperatures and elongation times were used: Ddit4: forward 5'-CTT CCT TGG TCC CTG GTT AC 3', reverse 5' -CGG TGG CTA TTG TCA GTT TT- $3^{\prime}, 56^{\circ} \mathrm{C}, 9 \mathrm{~s}$; Bhlhb2: forward 5'-GGG AGC AGA GTG GTA GTG AC-3', reverse 5'-TGG TGG GAT GAG ATA GAA GG-3', 56 ${ }^{\circ} \mathrm{C}, 12 \mathrm{~s}$; map3k1: forward 5'-GGC GTT TGT TCC CTG TAA 


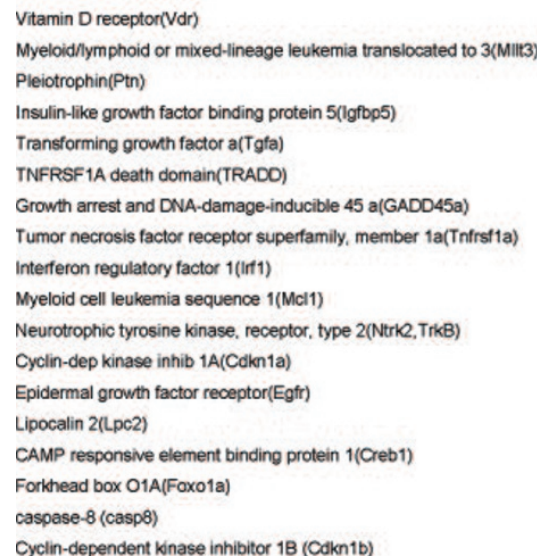

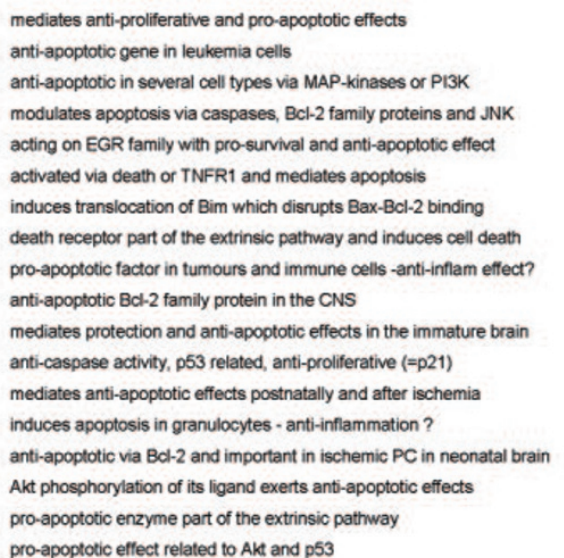

\begin{tabular}{|c|c|c|c|}
\hline $62^{*}$ & NM_017058 & $\downarrow 1.2$ & \\
\hline $63 *$ & BF549837 & 1.1.2 & \\
\hline 64 * & NM_017066 & $\downarrow 1.2$ & \\
\hline $65 * r)$ & BF399783 & 1.1.2 & \\
\hline $66^{*}$ & NM_012671 & 1.1 .2 & \\
\hline 67 *s) & $8 M 386846$ & $\downarrow 1.2$ & \\
\hline $68 * t)$ & NM_024127 & 1.1 .3 & \\
\hline$* u)$ & NM_013091 & A 1.3 & \\
\hline $\left.69{ }^{*} v\right)$ & NM_012591 & A 1.3 & \\
\hline $70 *$ w) & Al102618 & $\wedge 1.3$ & \\
\hline $71^{*}$ & BE 102996 & $\wedge 1.3$ & \\
\hline $\left.72{ }^{*} \mathrm{x}\right)$ & U24174 & 11.4 & $\uparrow 1.2$ \\
\hline $\left.66^{*} y\right)$ & M37394 & $\uparrow 1.4$ & \\
\hline $3,74 *$ & NM_130741 & $\wedge \mathbf{2 , 0}$ & $\uparrow 1.9$ \\
\hline $75 \mathrm{Z})$ & NM_031017 & A 1.2 & $\uparrow 1.2$ \\
\hline 76 *aa) & B1295511 & & 个 1.2 \\
\hline * & NM_022277 & & 个 1.2 \\
\hline 77 *bb) & NM_031762 & & 个 1.5 \\
\hline
\end{tabular}

\begin{abstract}
Genes related to apoptosis significantly different in PC vs. sham at $\mathrm{Oh}, 2 \mathrm{~h}, 8 \mathrm{~h}$ or $24 \mathrm{~h}$ are presented ( $\mathrm{t}$-test, FDR $0.05,1.2$ fold, B\&H correction). Fold-change is presented for genes that are significantly upregulated (red) or downregulated (green). References for Figure 1 are given in a separate reference list (available as supplemental material at $\mathrm{mw}$. pedresearch.com). Novel genes that have not previously been shown to be regulated after hypoxic PC in the brain are indicated by *. References are given for genes previously detected after PC. The combination of * and a reference indicates genes not previously shown to be regulated after PC in the brain but with previously published data supporting a role in PC.
\end{abstract}

Figure 1. (Continued)

AA-3', reverse 5'-GCC TTT GCC CTG TGT ATG TT-3', 60 $\mathrm{C}, 7 \mathrm{~s}$; Litaf: forward 5'-GAT CGT GAC CCA GTT GTC CT-3', reverse 5'-CGG GAG CAC TTG TCT ACC TC- $3^{\prime}, 60^{\circ} \mathrm{C}, 9 \mathrm{~s}$; Tnfrsfla: forward 5'-CTG AGT GAG ACG CAT TTC CA- ${ }^{\prime}$, reverse 5' CTG GAG GTA GGC ACA GCT TC-3', $60^{\circ} \mathrm{C}, 8 \mathrm{~s}$; Atf3: forward 5'-AGC CAA GGA TTC TCC GTT TT- $3^{\prime}$, reverse $5^{\prime}$-GGA CCG CAT CTC AAA ATA GC- $3^{\prime}, 62^{\circ} \mathrm{C}, 7 \mathrm{~s}$; Dusp1: forward 5'-TGA AGC AGA GGC GGA GTA TT-3', reverse 5'-TGA TGG GGC TTT GAA GGT AG- $3^{\prime}, 60^{\circ} \mathrm{C}, 9 \mathrm{~s}$; GAPDH: forward 5'-CTA CCC ACG GCA AGT TCA AC- $3^{\prime}$, reverse $5^{\prime}$-ACG CCA GTA GAC TCC ACG AC- $3^{\prime}, 58 \mathrm{C}^{\circ}$, $6 \mathrm{~s}$. Each sample was assayed in duplicate. Melting curve analysis was performed to ensure that only one product was amplified, and PCR samples were separated on $1.5 \%$ agarose gels for confirmation of product size. For quantification and for estimating amplification efficiency, a standard curve for each gene product was generated using increasing concentration of cDNA. Amplification transcripts were quantified using the appropriate relative standard curve and normalized against glyceraldehyde-3-phosphate dehydrogenase (GAPDH).

Confirmation of the neuroprotective effects by $P C$ in response to subsequent HI. Littermates of animals used for the microarray analysis ( $\mathrm{PC} n=44$; sham $n=36$ ) were subjected to an HI insult on PND 7 as previously described (3). In brief, animals were anesthetized with enflurane $(3.5 \%$ for induction and $1.5 \%$ for maintenance) in a mixture of oxygen and nitrogen. The left common carotid artery was cut between two 6-0 Prolene sutures. After anesthesia and surgery, the animals were returned to the dams and allowed $1 \mathrm{~h}$ of recovery. Thereafter, animals were exposed to $1 \mathrm{~h} 15 \mathrm{~min}$ of hypoxia in a humidified chamber at $36^{\circ} \mathrm{C}$ with $7.7 \%$ oxygen in nitrogen. After completion of the hypoxic interval, the animals were returned to their dams. On PND 14, animals were deeply anaesthetized (pentothal sodium, $50 \mathrm{mg} /$ $\mathrm{mL}$ ) and perfused intracranially with $0.9 \% \mathrm{NaCl}$ followed by $5 \%$ paraformaldehyde. Thereafter, brains were macroscopically scored for neuropathological outcome as previously described (3).

\section{RESULTS}

Changes in gene expression in sham controls between PND 6 and 7. Before analyzing the gene expression related to hypoxic PC, we analyzed the developmental changes over the 24-h experimental period in sham controls. A total of 3424 genes were significantly altered between PND 6 and 7 (ANOVA, BH FDR 0.05, 1.2-fold change) in sham controls. Cluster analysis (partitioning around medoids) revealed that most genes (1774) were down-regulated. Pairwise analysis ( $0 \mathrm{~h}$ versus $24 \mathrm{~h}$; $t$ test, BH FDR 0.05, 1.2-fold change) also showed that the majority of genes were down-regulated. According to functional ontology analysis, many of the regulated genes were, not unexpectedly, related to metabolism, cell communication, transport, cell growth/proliferation, differentiation, maturation and organization, neurogenesis, cell motility, and behavior. Generally, genes that were most developmentally regulated in the cerebral cortex between PND 6 and 7 were not the ones affected by PC hypoxia. However, the substantial number of developmentally regulated genes over the 24-h period in this study justified the use of pairwise analysis between PC hypoxia and separate precisely agematched controls that were drawn from the same litters at 0,2 , 8 , or $24 \mathrm{~h}$.

Transcriptome changes induced by PC: ontology analysis. Table 1 summarizes the number of genes that were significantly up- and down-regulated at $0,2,8$, and $24 \mathrm{~h}$ after PC ( $t$ test BH FDR 0.05, 1.2-fold change). Generally, there were more genes regulated at early (906 at $0 \mathrm{~h}$ and 927 at $2 \mathrm{~h}$ after PC) than at late (389 at $8 \mathrm{~h}$ and 114 at $24 \mathrm{~h}$ after PC) time points. Gene ontology (GO) analysis was performed with strict criteria including only those gene categories with $>10$ genes and with a $Z$ score less than -2 or $>2$. GO analysis revealed that at $0 \mathrm{~h}$ and $2 \mathrm{~h}$ after PC, up-regulated genes related to transcription, phosphorylation, and brain development (regulation of cell proliferation, cell/organelle organization and biogenesis, cell differentiation, organogenesis, neurogenesis, blood vessel development) were overrepresented, whereas up-regulated genes related to transport were underrepresented (Table 2). Genes involved in cell death/apoptosis or cell fate determination were overrepresented at 0 and $2 \mathrm{~h}$ after PC. Up-regulated signal transduction genes were also overrepresented, and pathway analysis indicated that many of these were part of the mitogen-activated protein kinase signaling, neuroactive ligand-receptor interaction, calcium signaling, cytokine-cytokine receptor interaction, and transforming growth factor $\beta$ (TGF- $\beta$ ) signaling pathways. A striking up-regulation of genes in the TGF- $\beta$ signaling pathway was observed ( $Z$ score 3.21) (not shown). At $8 \mathrm{~h}$ after PC, genes related to cell catabolism as well as cell stress (response to external stimulus, response to biotic stimulus, defense re- 
sponse) were up-regulated. The gene expression reaction to PC had subsided even further at $24 \mathrm{~h}$, and the limited number of genes that were ontogenetically classified were related to metabolism, cell growth and maintenance, response to external stimulus/stress, or cell communication (not shown).

In summary, ontology analysis at $0 \mathrm{~h}, 2 \mathrm{~h}$, and to some extent $8 \mathrm{~h}$ indicated that genes related to apoptotic cell death were overrepresented (Table 2). At $0 \mathrm{~h}$, genes involved in both positive and negative regulation of apoptosis were significantly up-regulated. However, at $2 \mathrm{~h}$, only antiapoptotic genes (e.g. Hspala, Bag3, Cebpb, Notch2, Bnip3, Tgfa) were upregulated ( $Z$ score 3.49 ), whereas some proapoptotic genes (e.g. TRADD, tial, id3, Lspl, vdr) were significantly downregulated, suggesting a shift toward an increased anti-/ proapototic balance.

PC-induced changes in apoptosis-related genes. To track genes that may specifically affect cellular vulnerability, we also individually scrutinized genes that were significantly altered at $0,2,8$, or $24 \mathrm{~h}$ ( $t$ test versus sham, BH FDR 0.05 , 1.2-fold change) and found that 77 genes related to apoptotic or nonapoptotic cell death were significantly affected (Fig. 1). Some of these genes were related to the Bcl-2 family, JNK pathway, trophic factors, PI3 kinase/Akt pathway, extrinsic pathway, caspase-cascade, or the p53 pathway (Fig. 2).

Real-time PCR. Seven genes (Ddit4, Bhlhb2, map3k1, Litaf, Tnfrsfla, Atf3, and Dusp1) were selected for confirmation as they represent examples of putatively anti- or proapoptotic genes (Table 3, refer to supplemental material online) that may contribute to PC protection. Six of these genes were significantly regulated according to the microarray results. There was a good agreement between the microarray and RTPCR results irrespective of the magnitude of the difference (1.2to 2.9-fold) and level of significance ( $p<0.05$ to $p<0.001$ ), supporting that the criteria ( $t$ test versus sham, BH FDR 0.05, 1.2-fold change) used in the present study are reasonable (Fig. 3).

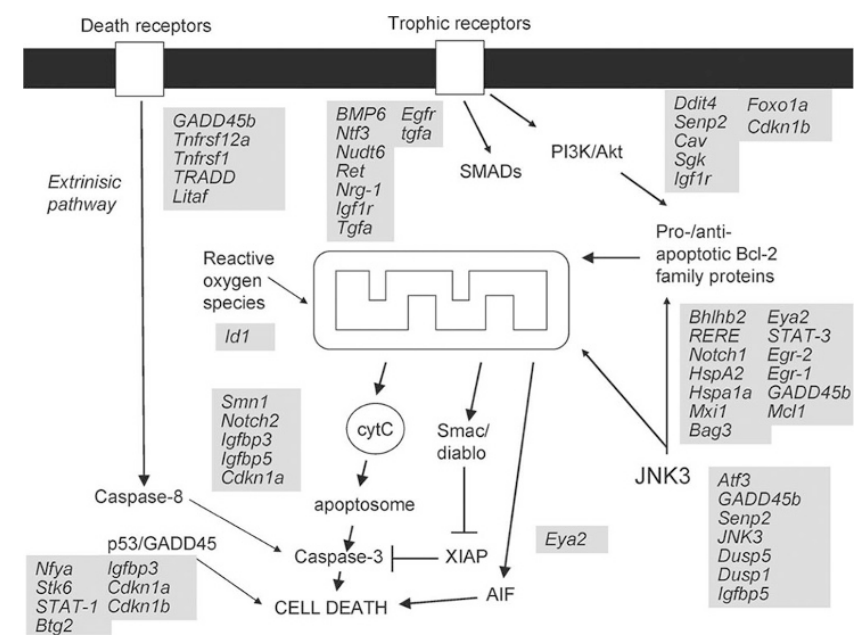

Figure 2. A summary of genes regulated by PC that are likely to be involved in intrinsic and extrinsic caspase-dependent as well as caspase-nondependent cell death process. Genes related to the trophic factors, PI3 K/Akt pathway, Bcl-2 family, JNK pathway, intrinsic caspase cascade, extrinsic pathway, p53-dependent apoptosis, reactive oxygen species, and noncaspase-dependent cell death appear in shaded areas.
One of the genes (Tnfrsfla) was not significantly regulated at $0 \mathrm{~h}$ after PC according to the microchip array analysis, which was also confirmed by RT-PCR. (Ddit4 (also called REDD1 and RTP801) is strongly induced in a hypoxia-inducible factor-1 (HIF-1)-dependent manner, is a downstream target in the PI3kinase-Akt pathway, and is believed to exert strong antiapoptotic affects and promote cancer growth (22). Bhlhb2 (also called DEC1 and STRA13) is highly expressed in colon carcinoma cells and appears to have a role in oncogenesis related to its antiapoptotic properties. It inhibits activation of caspases (caspase-3, -7, and -9) in the intrinsic pathway but not caspase-8 (23).

Confirmation of PC effect on CNS vulnerability. Littermates of the animals used for microarray analysis were also subjected to PC or sham PC on PND 6, followed by HI on PND 7. Macroscopic evaluation of these brains on PND 14 demonstrated a significant reduction of $\mathrm{HI}$ brain injury in animals pre-exposed to PC hypoxia $(0.8 \pm 0.2, n=44)$ in comparison with sham animals $(2.7 \pm 0.2, n=36, p<0.0001)$.

\section{DISCUSSION}

We found that, of the 31,000 genes/expressed sequence tags analyzed, more than 2000 genes were up- or down-regulated $0,2,8$, or $24 \mathrm{~h}$ after hypoxic PC using relatively strict statistical criteria. It is important to stress that the dynamics of the transcriptome (3424 genes changed significantly between PND 6 and 7) in the developing brain, which necessitated comparison with age-matched sham controls from the same litters. We also had an equal gender distribution in the two groups, as differences in gene expression between males and females during embryonic development have been reported (24). PC hypoxia up-regulated genes involved in cell communication, signal transduction, transcription, and phosphorylation. Genes involved in death/apoptosis or cell fate determination were overrepresented and as well as those related to brain development (differentiation, neurogenesis, organogenesis, blood vessel development). It is interesting that so many genes related to CNS development were modulated by PC hypoxia, and this response could of course be beneficial for repair and functional compensation after the insult. Conversely, it will be a challenge for the future to understand how the developing CNS manages to develop resistance to a subsequent insult, including altered expression of hundreds of development-related genes, without adversely interfering with the molecular program of brain development. Indeed, PC hypoxia seems to provide both functional and structural protection in the long term using this model (3) even though we do not know whether PC hypoxia (without a superimposed insult) affects neurofunctional behavior compared with sham controls. Both chronic hypoxia (25) and lipopolysaccharide (26) have also been shown to change the expression of genes related to brain development, but these exposures increase rather than decrease cerebral vulnerability. The ontology analysis revealed that genes related to apoptosis as a group were significantly regulated in response PC hypoxia, which is interesting in light of the key role of apoptotic mechanisms in immature brain injury (see introduction). However, only a minority of relevant genes are included in an ontology report; 

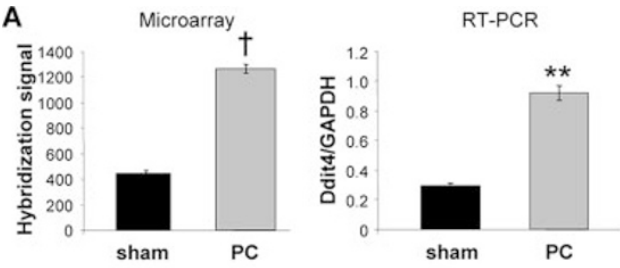

Fold change: 2.9

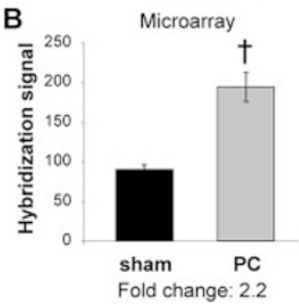

C
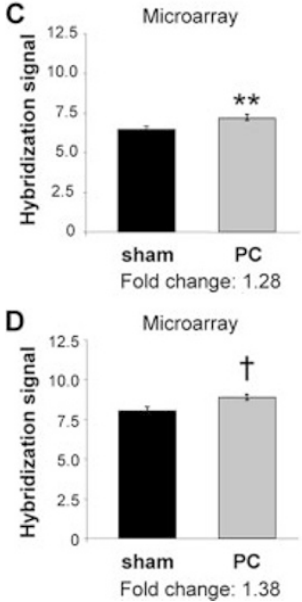
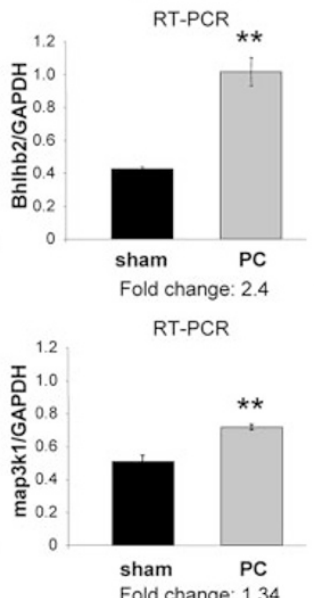

Fold change: 1.34

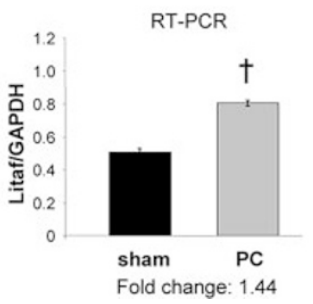

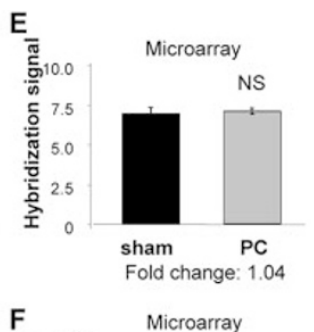
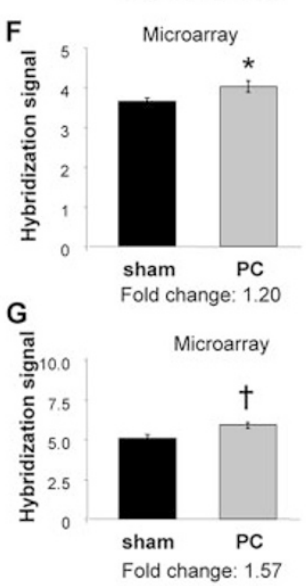

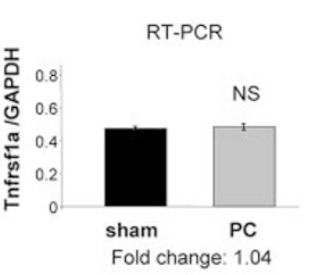

RT-PCR
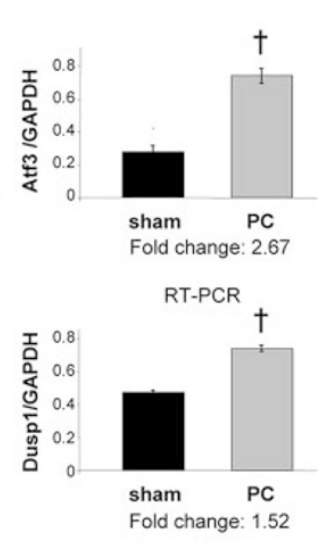

Figure 3. Results obtained by microarray [presented as mean $\log _{2}$ transformed hybridization signal values + standard error of the mean (SEM)] were confirmed by real-time PCR (mean + SEM transcript signals normalized against GAPDH) for Ddit4 (A), Bhlhb2 (B), map3k1 (C), Litaf $(D)$, Tnfrsfla $(E), \operatorname{Atf3}(F)$, and Duspl $(G)$ at $0 \mathrm{~h}$ after PC. The fold change for each gene were calculated based on delogged hybridization signal microchip and RTPCR results. $* p<0.05 ; * * p<0.01 ; \dagger p<$ 0.001 .

careful scrutiny of all genes that were significantly regulated revealed that 77 genes related to apoptotic mechanisms were significantly changed after PC hypoxia, most of them at 0 and $2 \mathrm{~h}$ and most of them not previously reported to be altered by $\mathrm{PC}$ in the immature brain. Many of these genes are involved in proliferation, cell cycle regulation, and differentiation as well as apoptosis, and, not seldom, both pro- and antiapoptotic effects have been reported. It is also important to point out that the direction of change in gene expression may not reflect the functional role of the protein, e.g. caspase-3 activity is increased after HI but the gene expression decreases (7). Nevertheless, there are many examples of predominantly antiapoptotic genes that were increased by hypoxic PC: Mtla, Ddit4, Bhlhb2, BMP6, ATF3, ntf3, GADD45b, Nudt6, Notchl/ Hes1, hspa2, hspala, Cav, Bag3, STAT3, Btg2, Nrg-1, Igflr, Cebpb, Sgk, Hmoxl, Mllt3, Ptn, Tgfa/Egfr, Mcll, TrkB, cdknla, crebl, and Foxola and a number of proapoptotic genes that are down-regulated (Nfya, Stk17b, Gpr37, TRADD). There were also changes in some genes (tff2, Smnl, Csrp2, Timp3, Zyx, Pkn1, Tnfrsf12a/tnfrsfla, Eya2, Gadd45a, Egr1/2, Irf1, Lpc2, casp8, cdkn1b) that may seem paradoxical and difficult to reconcile with decreased cellular vulnerability. Some of these genes have anti-inflammatory properties (Timp3, Zyx, Pkn1, Tnfrsf12a/la, Lpc2, casp8, Irf1) and may not be involved in neuronal or oligodendroglial apoptosis but could be beneficial by promoting apoptosis of inflammatory cells, limiting damage caused by these cells. In addition, moderate activation of proapototic systems such as the caspases could be a mechanism whereby protective heat shock proteins are induced in response to PC in the adult brain (27). There were also a number of genes up- or down-regulated that are likely to be involved in apoptosis (e.g. RERE, CREM, Senp2, Tle3, Stk6), but, based on the current literature, there is insufficient information to decide whether the change in gene expression is likely to increase or decrease cell survival. Several of these findings deserve to be studied in more detail because they may be important molecular events in the development of cerebral resistance. As many as 13 genes related to the $\mathrm{Bcl}-2$ family of proteins that have not been previously reported to be affected by PC were regulated in this study (Fig. 1). This agrees with a few studies demonstrating that $\mathrm{Bcl}-2$ proteins are important in HI $(18,28,29)$, and ceramide "PClike" protection in the neonatal setting was accompanied by up-regulation of both Bcl-2 and Bcl-xL (30). JNK3 was recently discovered to exert proapoptotic effects in HI (31). Several genes related to this pathway (Atf3, Map3k1, Mapk10/ $J n k 3, J u n b)$ were altered by PC. Given that this pathway is activated through phosphorylation, it is interesting that the phosphatases dusp1 and dusp6 were both up-regulated, which may contribute to inactivation of Jnk3, p38, and other kinases. The role of this family of phosphatases in cerebral vulnerability needs to be addressed in the future.

Nrg-1, Tgf1a, and Egfr were all up-regulated after hypoxic PC, which has not previously been shown. Neuregulins and 
Tgf1a both activate the epidermal growth factor family (Egfr, ErbB) of receptors and play important role in proliferation, migration, and differentiation of neuronal and glial cells; they also enhance survival and inhibit apoptosis of postmitotic neurons (32). Nrg-1 exerts a neuroprotective effect in the adult brain after stroke, which may be due to its antiapoptotic and anti-inflammatory properties (33). Tgf1a has also been shown to reduce ischemic brain injury in adults, likely via nonvascular neuronal actions (34). Our present results implicate the epidermal growth factor receptor family in regulating the vulnerability of the developing brain to injury, which needs to be studied in more detail.

The PI3 K/Akt pathway has been shown to be important for attenuation of apoptotic DNA fragmentation after ischemia in the adult brain (35) and is essential for ischemic PC in the liver (36). This pathway is also activated in the developing CNS after HI (37) and PI3 K/Akt is likely to partly explain the neuroprotective effect of insulinlike growth factor I (Igf-I) and the secretagogue hexarelin (37-39) even though the role of this system is not fully understood. We currently found that 16 genes directly or indirectly related to PI3 K/Akt were regulated in response to hypoxic PC (Figs. 1 and 2) suggesting that preservation of Akt activity may contribute to CNS tolerance after hypoxia.

Acknowledgments. The authors thank Anna-Lena Leverin and Eva Cambert for excellent technical assistance.

\section{REFERENCES}

1. Johnston MV, Nakajima W, Hagberg H 2002 Mechanisms of hypoxic neurodegeneration in the developing brain. Neuroscientist 8:212-220

2. Gidday JM, Fitzgibbons JC, Shah AR, Park TS 1994 Neuroprotection from ischemic brain injury by hypoxic preconditioning in the neonatal rat. Neurosci Lett 168:221224

3. Gustavsson M, Anderson MF, Mallard C, Hagberg H 2005 Hypoxic preconditioning confers long-term reduction of brain injury and improvement of neurological ability in immature rats. Pediatr Res 57:305-309

4. Barone FC, White RF, Spera PA, Ellison J, Currie RW, Wang X, Feuerstein GZ 1998 Ischemic preconditioning and brain tolerance: temporal histological and functional outcomes, protein synthesis requirement, and interleukin-1 receptor antagonist and early gene expression. Stroke 29:1937-1950

5. Johnston MV 2005 Gene expression profiling: a new tool for pediatric neurology? Curr Opin Neurol 18:89-90

6. Bernaudin M, Tang Y, Reilly M, Petit E, Sharp FR 2002 Brain genomic response following hypoxia and re-oxygenation in the neonatal rat. Identification of genes that might contribute to hypoxia-induced ischemic tolerance. J Biol Chem 277:39728 39738

7. Blomgren K, Zhu C, Wang X, Karlsson JO, Leverin AL, Bahr BA, Mallard C, Hagberg H 2001 Synergistic activation of caspase-3 by m-calpain after neonatal hypoxia-ischemia: a mechanism of "pathological apoptosis"? J Biol Chem 276:10191-10198

8. Ota K, Yakovlev AG, Itaya A, Kameoka M, Tanaka Y, Yoshihara K 2002 Alteration of apoptotic protease-activating factor-1 (APAF-1)-dependent apoptotic pathway during development of rat brain and liver. J Biochem (Tokyo) 131:131-135

9. Merry DE, Veis DJ, Hickey WF, Korsmeyer SJ 1994 Bcl-2 protein expression is widespread in the developing nervous system and retained in the adult PNS. Development 120:301-311

10. Vekrellis K, McCarthy MJ, Watson A, Whitfield J, Rubin LL, Ham J 1997 Bax promotes neuronal cell death and is downregulated during the development of the nervous system. Development 124:1239-1249

11. Cheng Y, Deshmukh M, D'Costa A, Demaro JA, Gidday JM, Shah A, Jacquin MF, Johnson EM, Holtzman DM 1998 Caspase inhibitor affords neuroprotection with delayed administration in a rat model of neonatal hypoxic-ischemic brain injury. J Clin Invest 101:1992-1999

12. Nakajima W, Ishida A, Lange MS, Gabrielson KL, Wilson MA, Martin LJ, Blue ME, Johnston MV 2000 Apoptosis has a prolonged role in the neurodegeneration after hypoxic ischemia in the newborn rat. J Neurosci 20:7994-8004
13. Wang X, Karlsson JO, Zhu C, Bahr BA, Hagberg H, Blomgren K 2001 Caspase-3 activation after neonatal rat cerebral hypoxia-ischemia. Biol Neonate 79:172-179

14. Zhu C, Qiu L, Wang X, Hallin U, Cande C, Kroemer G, Hagberg H, Blomgren K 2003 Involvement of apoptosis-inducing factor in neuronal death after hypoxiaischemia in the neonatal rat brain. J Neurochem 86:306-317

15. Zhu C, Wang X, Hagberg H, Blomgren K 2000 Correlation between caspase-3 activation and three different markers of DNA damage in neonatal cerebral hypoxia ischemia. J Neurochem 75:819-829

16. Wang X, Zhu C, Hagberg H, Korhonen L, Sandberg M, Lindholm D, Blomgren K 2004 X-linked inhibitor of apoptosis (XIAP) protein protects against caspase activation and tissue loss after neonatal hypoxia-ischemia. Neurobiol Dis 16:179-189

17. Gill R, Soriano M, Blomgren K, Hagberg H, Wybrecht R, Miss MT, Hoefer S, Adam G, Niederhauser O, Kemp JA, Loetscher H 2002 Role of caspase-3 activation in cerebral ischemia-induced neurodegeneration in adult and neonatal brain. J Cereb Blood Flow Metab 22:420-430

18. Northington FJ, Ferriero DM, Flock DL, Martin LJ 2001 Delayed neurodegeneration in neonatal rat thalamus after hypoxia-ischemia is apoptosis. J Neurosci 21:19311938

19. Bolstad BM, Irizarry RA, Astrand M, Speed TP 2003 A comparison of normalization methods for high density oligonucleotide array data based on variance and bias. Bioinformatics 19:185-193

20. Irizarry RA, Bolstad BM, Collin F, Cope LM, Hobbs B, Speed TP 2003 Summaries of Affymetrix GeneChip probe level data. Nucleic Acids Res 31:e15

21. Benjamini Y, Hochberg Y 1995 Controlling the false discovery rate: a practical and powerful approach to multiple testing. J R Stat Soc B 57:289-300

22. Schwarzer R, Tondera D, Arnold W, Giese K, Klippel A, Kaufmann J 2005 REDD1 integrates hypoxia-mediated survival signaling downstream of phosphatidylinositol 3 kinase. Oncogene 24:1138-1149

23. Li Y, Zhang H, Xie M, Hu M, Ge S, Yang D, Wan Y, Yan B 2002 Abundant expression of Dec1/stra13/sharp2 in colon carcinoma: its antagonizing role in serum deprivation induced apoptosis and selective inhibition of procaspase activation. Biochem J 367:413-422

24. Yonehara K, Suzuki M, Nishihara M 2002 Sex-related differences in gene expression in neonatal rat hypothalamus assessed by cDNA microarray analysis. Endocr $\mathrm{J}$ 49:131-137

25. Curristin SM, Cao A, Stewart WB, Zhang H, Madri JA, Morrow JS, Ment LR 2002 Disrupted synaptic development in the hypoxic newborn brain. Proc Natl Acad Sci U S A 99:15729-15734

26. Eklind S, Hagberg H, Wang X, Sävman K, Hedtjärn M, Mallard C 2006 Effect of lipopolysaccharide on global gene expression in the immature rat brain. Pediatr Res 60:161-168

27. McLaughlin B, Hartnett KA, Erhardt JA, Legos JJ, White RF, Barone FC, Aizenman E 2003 Caspase 3 activation is essential for neuroprotection in preconditioning. Proc Natl Acad Sci U S A 100:715-720

28. Gibson ME, Han BH, Choi J, Knudson CM, Korsmeyer SJ, Parsadanian M, Holtzman DM 2001 BAX contributes to apoptotic-like death following neonatal hypoxia-ischemia: evidence for distinct apoptosis pathways. Mol Med 7:644-655

29. Parsadanian AS, Cheng Y, Keller-Peck CR, Holtzman DM, Snider WD 1998 Bcl xL is an antiapoptotic regulator for postnatal CNS neurons. J Neurosci 18:1009-1019

30. Chen Y, Ginis I, Hallenbeck JM 2001 The protective effect of ceramide in immature rat brain hypoxia-ischemia involves up-regulation of bcl-2 and reduction of TUNEL positive cells. J Cereb Blood Flow Metab 21:34-40

31. Kuan CY, Whitmarsh AJ, Yang DD, Liao G, Schloemer AJ, Dong C, Bao J, Banasiak KJ, Haddad GG, Flavell RA, Davis RJ, Rakic P 2003 A critical role of neural-specific JNK3 for ischemic apoptosis. Proc Natl Acad Sci U S A 100:1518415189

32. Xian CJ, Zhou XF 1999 Roles of transforming growth factor-alpha and related molecules in the nervous system. Mol Neurobiol 20:157-183

33. Unoki M, Nakamura Y 2003 EGR2 induces apoptosis in various cancer cell lines by direct transactivation of BNIP3L and BAK. Oncogene 22:2172-2185

34. Justicia C, Perez-Asensio FJ, Burguete MC, Salom JB, Planas AM 2001 Administration of transforming growth factor-alpha reduces infarct volume after transient focal cerebral ischemia in the rat. J Cereb Blood Flow Metab 21:1097-1104

35. Noshita N, Lewen A, Sugawara T, Chan PH 2001 Evidence of phosphorylation of Akt and neuronal survival after transient focal cerebral ischemia in mice. J Cereb Blood Flow Metab 21:1442-1450

36. Izuishi K, Tsung A, Hossain MA, Fujiwara M, Wakabayashi H, Masaki T, Billiar TR, Maeta H 2006 Ischemic preconditioning of the murine liver protects through the Akt kinase pathway. Hepatology 44:573-580

37. Brywe KG, Mallard C, Gustavsson M, Hedtjarn M, Leverin AL, Wang X, Blomgren K, Isgaard J, Hagberg H 2005 IGF-I neuroprotection in the immature brain after hypoxia-ischemia, involvement of Akt and GSK3beta? Eur J Neurosci 21:14891502

38. Cao Y, Gunn AJ, Bennet L, Wu D, George S, Gluckman PD, Shao XM, Guan J 2003 Insulin-like growth factor (IGF)-1 suppresses oligodendrocyte caspase-3 activation and increases glial proliferation after ischemia in near-term fetal sheep. J Cereb Blood Flow Metab 23:739-747

39. Brywe KG, Leverin AL, Gustavsson M, Mallard C, Granata R, Destefanis S, Volante M, Hagberg H, Ghigo E, Isgaard J 2005 Growth hormone-releasing peptide hexarelin reduces neonatal brain injury and alters Akt/glycogen synthase kinase-3beta phosphorylation. Endocrinology 146:4665-4672 